\title{
GLOBAL BLOCK-SIMILARITY AND POLE ASSIGNMENT OF CLASS $C^{P}$
}

\author{
JOSEP FERRER* AND FERRAN PUERTA ${ }^{\dagger}$
}

\begin{abstract}
Starting from the existence of a $C^{p}$-basis for any $C^{p}$-family of subspaces having constant dimension, we construct a Brunovsky basis of class $C^{p}$ for a $C^{p}$-family of pairs of matrices having constant Brunovsky type. We derive a global pole assignment theorem for such kind of pairs. In all the cases we assume that the manifold of parameters is contractible.
\end{abstract}

Key words. Block-similarity, Brunovsky, pole assignment, principal bundles, families of subspaces

AMS subject classifications. $93 \mathrm{~B}, 53 \mathrm{C}$.

1. Introduction. There is a large literature concerning parametrized families of linear systems. "Most results attempt in essence to establish local-global principles: does pointwise solvability imply the existence of nicely parametrized solution?" [17].

In particular, there is also a large literature concerning the problems dealt with in this paper: global reduction to the canonical form, and global pole assignment. Both problems have been widely studied for pointwise controllable families. One of the aims of this paper is to present a generalisation to families non-necessarily pointwise controllable.

For a general introduction to families of linear systems, see for example [13]. There, some problems that justify the study of families of systems are presented, and one tackles the classification of families (fine moduli spaces), the existence of global canonical forms and some others. In fact, that work deals with a more general class of families of linear systems, in terms of bundles over the space of parameters, which includes the parametrized ones.

An alternative generalization is the consideration of systems over rings, this is to say, pairs of matrices with entries on a commutative ring. The particular case of parametrized families arises when rings of functions defined in the space of parameters are considered. See [15] and [2] for a general introduction, and [17] for a survey and many references, mainly centered on control and stabilization problems.

The Swan theorem connects these approaches to local-global problems by means of the correspondence between vector bundles and projective modules.

Let us see in more detail the problems explicitely studied in this paper. The central one is theorem 6 , in 3.4: the existence of a differentiable reduction to the Brunovsky form (or equivalently, the existence of a differentiable Brunovsky basis) for a differentiable family of pairs of matrices having constant Brunovsky type, provided that the manifold of parameters is contractible.

For pointwise completely controllable families, the result is classical in the continuous case [2] or for polynomial rings [18]. It is also well known in the differentiable case and used in the literature without explicit reference (see for example [14] and

\footnotetext{
*Departament de Matemtica Aplicada I, ETSEIB-UPC Avda. Diagonal, 647, Barcelona-08028. Spain (ferrer@ma1.upc.es).

$\dagger$ Departament de Matemtica Aplicada I, ETSEIB-UPC Avda. Diagonal, 647, Barcelona-08028. Spain (puerta@ma1.upc.es).
} 
[17]). On the other hand, the global reduction to the Jordan form for a family of square matrices has been more recently studied by [4], [8] and [5].

However, the extension to the general case starting on the above "extreme" cases (controllable, Jordan) does not seem trivial in spite of the global splitting in [13] (11.3.1) because this is not a direct split.

In fact, this obstruction is not surprising because it appears in other problems concerning parametrized families of systems. For example, the construction of a versal deformation has been solved in [1] for square matrices and in [18] for pointwise controllable pairs of matrices. But the general case does not derive from them (see $[6])$.

Secondly, we study the global pole assignment of parametrized families, which has been widely studied (see for example [17]), always under the hypothesis of pointwise controllability. The problem is solved en [2] and [16] by means of algebro-geometric and algebraic techniques respectively, provided constant controllability indices. Other conditions are considered, for example, in [19] (constant rank of $B$, ring controllability) or in [12] (one-dimensional manifold of parameters).

Here the general result for non-necessarily pointwise controllable families (theorem 7, in (4)) is derived as an application of the previous one about global reduction to Brunovsky form. By means of it, the proof in [9] of pole assignment for a pair of matrices (non-necessarily controllable) can be immediately translated into a differentiable family of pairs of matrices having constant Brunovsky type.

Going back to the central result about global reduction to the Brunovsky form, our technique consists essentially in extending the construction in the constant case to the parametrized one by means of the theorem 2, in (1): the existence of global differentiable bases for a differentiable family of subspaces having constant dimension, parametrized over a contractible manifold.

A classical and fundamental reference for this basic tool in the continuous case is [10]. In fact, it proves by means of the method of cocycles a generalization for operator valued functions defined on a contractible manifold, provided it is compact. In our case of matrix valued differentiable functions on a contractible manifold nonnecessarily compact, it is used for example in [13], and it is explicitely presented in [20] (remark after 2.5).

For the continuous case, the key point is [11] (3.4.8) about the triviality of bundles over contractible spaces. Then, the smooth case follows by means of approximation theorems. Alternatively, we have checked that the proof of this key point in [11] for the continuous case can be adapted to the differentiable one.

Notice that the hypothesis that the manifold of parameters must be contractible is only used for prop.A to be verified. Then, all the machinery works under others conditions whenever this prop.A holds. For example, under the conditions of [11] (8.1.2).

This technique for extending the local case to the parametrized one is possible if we have a geometrical description of the construction in the constant case (in terms of kernels, supplementary subspaces,...). In this case, we use the description of a Brunovsky basis obtained in [7], as a basis of the global space adapted to an increasing chain of certain subspaces.

The minimum subspace of this chain corresponds to the uncontrollable subsystem, 
whose Brunovsky bases are in fact Jordan bases. For this step, we remark that in [5] the same technique has been used for the case of square matrices having constant Jordan type, by means of the usual description of Jordan bases as adapted to an increasing chain of kernels.

Notice that this method does not need further references to algebro-geometric or algebraic techniques, but only the prop. A and the standard machinery for the constant case. As one of the referees has pointed about, this presentation seems more accessible to engineers.

Moreover, our approach has some connections with the one in [3]. There, the control properties of a pair of matrices $(A, B)$ are studied by means of the geometric properties of the curve $\gamma: \mathbb{R} \longrightarrow G r_{s, n}, \gamma(\tau)=\operatorname{Im}\left(e^{A \tau} B\right)$. Here, we do not consider a pair $(A, B)$, but a $C^{p}$-family of pairs $(A(t), B(t)), t \in M$, having constant Brunovsky type.Then, instead of a curve in $G r_{s, n}$, we could consider a multi-parametrized mapping $\Gamma: M \times \mathbb{R} \longrightarrow G r_{s, n}, \Gamma(t, \tau)=\operatorname{Im}\left(E^{A(t) \tau} B(\tau)\right)$. It can be expected that some control properties of the family should be related to the geometric properties of $\Gamma$.

In (1) and (2) we recall, respectively, the basic facts about differentiable families of subspaces and about global similarity of square matrices which we will use in the sequel. See [5] for more details. (3) is devoted to the proof of the main theorem 6. And (4) contains the application to global pole assignment.

Throughout the paper $K$ denotes $\mathbb{R}$ or $\mathbb{C},\left(e_{1}, \ldots, e_{n}\right)$ the standard basis of $K^{n}$, and $G r_{k, n}$ the set of $k$-dimensional subspaces of $K^{n}$. If $v_{1}, \ldots, v_{s}$ are vectors of $K^{n}$, then $\left[v_{1}, \ldots, v_{s}\right]$ will denote the subspace spanned by them.

We write $M_{n \times k}(K)$ for the vector space of $(n \times k)$-matrices with entries in $K$, $M_{n \times k}^{*}$ the open subset formed by the matrices $A \in M_{n \times k}(K)$ having rank $k(\leq n)$, and $G l(n)$ the linear group of non-singular matrices of $M_{n}(K)$. If $A \in M_{n \times m}(K)$, we also denote by $A$ the linear map from $K^{m}$ to $K^{n}$ defined by $\left(x_{1}, \ldots, x_{m}\right) \longrightarrow$ $\left(x_{1}, \ldots, x_{m}\right) A^{t}$, where $A^{t}$ is the transpose matrix of $A$. Id will denote the identity mapping, and $I d_{k}$ the identity $k$-matrix.

By a differentiable manifold we mean a $C^{p}$-manifold, $1 \leq p \leq \infty$. In all the paper $M$ will be a differentiable manifold. In the same way, by a differentiable map between two of such manifolds we mean a $C^{p}$-morphism.

We are grateful to the referees for their careful revision and their valuable suggestions.

2. Differentiable families of subspaces. In $G r_{k, n}$ the usual topology and differentiable structure are considered. With them, $G r_{k, n}$ is a compact homogeneous manifold. This topology is equivalent to the one induced by the gap metric [9]. A proof of this equivalence is given in [5].

By a family of $k$-subspaces of $K^{n}$ parametrized on $M$ we mean a map $\mathcal{L}$ : $M \longrightarrow G r_{k, n}$. If it is differentiable, we write $\mathcal{L} \in C^{p}\left(M, G r_{k, n}\right)$. The existence of "differentiable local basis" is a useful criterium for the differentiability of a family of subspaces. It follows immediately from the local triviality of a bundle:

Proposition 2.1. Let $M$ be a differentiable manifold, and $\mathcal{L}: M \longrightarrow G r_{k, n}$ a family of $k$-subspaces parametrized on $M$. Then, $\mathcal{L}$ is differentiable if and only if: for every $t_{0} \in M$ there is an open neighbourhood $W_{t_{0}}$ of $t_{0}$ in $M$, and $k$ maps 
$v_{i} \in C^{p}\left(W_{t_{0}}, K^{n}\right), 1 \leq i \leq k$, such that $\left\{v_{1}(t), \ldots, v_{k}(t)\right\}$ is a basis of $\mathcal{L}(t)$, for all $t \in W_{t_{0}}$.

For example, if $\mathcal{L} \in C^{p}\left(M, G r_{k, n}\right)$, then $\mathcal{L}^{\perp} \in C^{p}\left(M, G r_{n-k, n}\right)$, where $\mathcal{L}^{\perp}(t)=$ $\mathcal{L}(t)^{\perp}$. Also, if $A \in C^{p}\left(M, M_{n \times m}(K)\right)$ with rank $A(t)=k$, for all $t \in M$, then $\operatorname{Im}$ $A \in C^{p}\left(M, G r_{k, n}\right)$ and $\operatorname{Ker} A \in C^{p}\left(M, G r_{n-k, n}\right)$.

Let us assume that $M$ is contractible. As we have remarked in the introduction, a basic tool in our technique is the existence of a "differentiable global basis" (in fact, we will use the corollary):

THeOREM 2.2 ((see the introduction for the references)). Let $M$ be a contractible manifold, and $\mathcal{L} \in C^{p}\left(M, G r_{k, n}\right)$ a differentiable family of $k$-subspaces parametrized on $M$. Then there exist $k$ maps $v_{i} \in C^{p}\left(M, K^{n}\right), 1 \leq i \leq k$, such that $\left\{v_{1}(t), \ldots, v_{k}(t)\right\}$ is a basis of $\mathcal{L}(t)$ for every $t \in M$.

Corollary 2.3. Let $M$ be a contractible manifold, and $\mathcal{L}_{i} \in C^{p}\left(M, G r_{k_{i}, n}\right)$, $1 \leq i \leq 2$, such that $\mathcal{L}_{1}(t) \subset \mathcal{L}_{2}(t), \quad$ for all $t \in M$. Then there exist $v_{1}, \ldots, v_{k} \in$ $C^{p}\left(M, K^{n}\right), k=k_{2}-k_{1}$, such that

$$
\mathcal{L}_{2}(t)=\mathcal{L}_{1}(t) \oplus\left[v_{1}(t), \ldots, v_{k}(t)\right], \quad \text { for all } \quad t \in M
$$

3. Global similarity of class $C^{p}$. As we have said in the introduction, the first step in the proof of theorem 6 about block-similarity of class $C^{p}$ is the already known analogous result concerning similarity. Let $M$ be a differentiable manifold, and $A \in C^{p}\left(M, M_{n}(\mathbb{C})\right)$; that is to say, $\mathrm{A}(t)$ is a family of $n$-square complex matrices, parametrized by $t \in M$, and of class $C^{p}$. The family $A(t)$ is said to have constant Jordan type if the number of distinct eigenvalues and the list of the sizes of the Jordan blocks corresponding to different eigenvalues are independent of $t$.

Proposition 3.1. Let $M$ be a simply connected manifold, and $A \in C^{p}\left(M, M_{n}(\mathbb{C})\right)$ having constant Jordan type. Then:

(i) there exist $\lambda_{1}, \ldots, \lambda_{q} \in C^{p}(M, \mathbb{C})$ such that $\lambda_{1}(t), \ldots, \lambda_{q}(t)$ are the $q$ distinct eigenvalues of $A(t)$, for every $t \in M$.

(ii) the respective algebraic multiplicities $m_{1}, \ldots, m_{q}$ of these eigenvalues are constant.

Theorem 3.2. Let $M$ be a contractible manifold, and $A \in C^{p}\left(M, M_{n}(\mathbb{C})\right)$ having constant Jordan type. Then, there exists $S \in C^{p}(M, G l(n))$ such that $S(t)^{-1} A(t) S(t)$ is a Jordan matrix, for all $t \in M$.

\section{Global block-similarity of class $C^{p}$.}

4.1. We recall some basic properties of the block-similarity of pairs of matrices. Let us consider pairs of matrices $(A \quad B)$, where $A \in M_{n}(\mathbb{C})$ and $B \in M_{n \times m}(\mathbb{C})$. Two of such pairs $\left(\begin{array}{ll}A & B\end{array}\right)$ and $\left(\begin{array}{ll}A^{\prime} & B^{\prime}\end{array}\right)$ are called block-similar if there are complex matrices $S \in G l(n), T \in G l(m)$ and $C \in M_{m \times n}$ such that: $A^{\prime}=S^{-1}\left(A+B C S^{-1}\right) S$, $B^{\prime}=S^{-1} B T$, or equivalently

$$
\left(\begin{array}{ll}
A^{\prime} & B^{\prime}
\end{array}\right)=S^{-1}\left(\begin{array}{ll}
A & B
\end{array}\right)\left(\begin{array}{cc}
S & 0 \\
C & T
\end{array}\right)
$$


Every pair $\left(\begin{array}{ll}A & B\end{array}\right)$ is block-similar to its so-called Brunovsky form

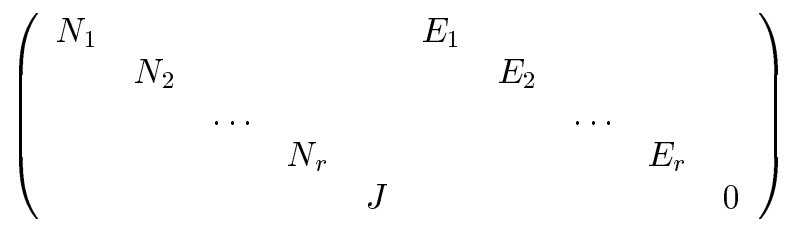

where $k_{1} \geq \ldots \geq k_{r}, N_{i}$ is a nilpotent $k_{i}$-matrix, $E_{i}$ is the column $\left(k_{i} \times 1\right)$-matrix transpose of $(0 \ldots 0 \quad 1)$, and $J$ is a Jordan matrix. This canonical form is unique, up to permutations of the Jordan blocks in $J$. In particular, $r=\operatorname{rank} B$, and $s \equiv$ $k_{1}+\ldots+k_{r}=\operatorname{rank}\left(B A B \ldots A^{n-1} B\right)$.

Numbers $k_{1}, \ldots, k_{r}$ are called the controllability indices of $\left(\begin{array}{ll}A & B\end{array}\right)$. Although the Jordan matrix $J$ is not uniquely determined, its similarity invariants are well defined: we will refer to them as the Jordan invariants of the pair $\left(\begin{array}{ll}A & B\end{array}\right)$. In particular, the eigenvalues of the pair $\left(\begin{array}{ll}A & B\end{array}\right)$ are those of $J$.

Two pairs are block-similar if and only if they have the same Brunovsky form. Thus, the controllability indices and the Jordan invariants of the pair form a complete family of invariants for the block-similarity.

Analogous considerations are valid for pairs of matrices of the form $\left(\begin{array}{l}A \\ B\end{array}\right)$. By duality, both constructions are equivalent. In particular, the controllability indices and the Jordan invariant of a pair $\left(\begin{array}{ll}A & B\end{array}\right)$ coincide with those of its transpose $\left(\begin{array}{l}A^{t} \\ B^{t}\end{array}\right)$.

4.2. We shall follow the method in [7] for the construction of Brunovsky bases. We recall that, up to reordering, a Brunovsky basis of a pair $\left(\begin{array}{l}A \\ B\end{array}\right)$ is obtained there by successive extensions in the chain of subspaces

$$
Y_{n}=\ldots=Y_{N} \subset Y_{N-1} \subset Y_{N-2} \subset \ldots \subset Y_{2} \subset Y_{1} \subset Y_{0} \equiv \mathbb{C}^{n} \subset Y_{-1} \equiv \mathbb{C}^{n+m}
$$

(the inclusions are strict) where

$$
Y_{i}=\operatorname{Ker}\left(\begin{array}{l}
B \\
B A \\
\ldots \\
B A^{i-1}
\end{array}\right), \quad 1 \leq i \leq n .
$$

One verifies that $A\left(Y_{N}\right) \subset Y_{N}$. And for $0 \leq i \leq N-1$, if $\bar{Y}_{i}$ is any complementary subspace to $Y_{i+1}$ in $Y_{i}$, this is to say $Y_{i}=Y_{i+1} \oplus \bar{Y}_{i}$, then: $A$ is injective on $\bar{Y}_{i}$; $A\left(\bar{Y}_{i}\right) \subset Y_{i-1} ; A\left(\bar{Y}_{i}\right) \cap Y_{i}=\{0\}$.

Moreover, in this description the controllability indices are characterized as the conjugate partition of the one formed by the differences $\operatorname{dim} Y_{i}-\operatorname{dim} Y_{i+1}$, and the Jordan invariants of the pair $\left(\begin{array}{l}A \\ B\end{array}\right)$ are those of the endomorphism $A \mid: Y_{N} \longrightarrow Y_{N}$, defined as the restriction of $A$. In particular, $k_{1}=N, r=n-\operatorname{dim} Y_{1}$, and $s=$ $n-\operatorname{dim} Y_{N}$.

4.3. Now let us consider families of pairs of matrices. Let $M$ be a differentiable manifold , and $\left(\begin{array}{ll}A & B\end{array}\right) \in C^{p}\left(M, M_{n \times(n+m)}(\mathbb{C})\right)$, that is to say, $(A(t) B(t))$ is a family of pairs of matrices, where $A \in C^{p}\left(M, M_{n}(\mathbb{C})\right)$ and $B \in C^{p}\left(M, M_{n \times m}(\mathbb{C})\right)$. We say that the family $(A(t) B(t))$ has constant Brunovsky type, if:

(i) the controllability indices are constant 
(ii) the Jordan invariants have constant type

Notice that, with regard to the description in 3.2 , it is equivalent to saying that:

(i') the dimensions of the subspaces $Y_{i}(t)$ are independent of $t$

(ii') the family of endomorphisms $A(t) \mid: Y_{N}(t) \longrightarrow Y_{N}(t)$ has constant Jordan type

4.4. For such a differentiable family $(A(t) \quad B(t))$ having constant Brunovsky type, the question lies in the construction of a Brunovsky basis depending differentiably on $t \in M$, provided that $M$ is contractible.

TheOREM 4.1. Let $M$ be a contractible manifold, and $(A B) \in C^{p}\left(M, M_{n \times(n+m)}(\mathbb{C})\right)$ a differentiable family of pairs of matrices having constant Brunovsky type. Then, there exist $S \in C^{p}(M, G l(n)), T \in C^{p}(M, G l(m))$ and $C \in C^{p}\left(M, M_{m \times n}(\mathbb{C})\right)$ such that

$$
S(t)^{-1}(A(t) \quad B(t))\left(\begin{array}{cc}
S(t) & 0 \\
C(t) & T(t)
\end{array}\right)
$$

is a Brunovsky matrix, for all $t \in M$.

Proof. Our aim is to adapt to parametrized pairs the construction relative to a constant pair $\left(\begin{array}{ll}A & B\end{array}\right)$ sketched in 3.2. In fact, as it was the case there, we shall deal with pairs of the form $\left(\begin{array}{l}A(t) \\ B(t)\end{array}\right)$. In order to do so, we shall consider the chain of subspaces

$$
Y_{n}(t)=\ldots=Y_{N}(t) \subset Y_{N-1}(t) \subset \ldots \subset Y_{1}(t) \subset Y_{0}(t) \equiv \mathbb{C}^{n} \subset \mathbb{C}^{n+m}
$$

where

$$
Y_{i}(t)=\operatorname{Ker}\left(\begin{array}{l}
B(t) \\
B(t) A(t) \\
\ldots \\
B(t) A(t)^{i-1}
\end{array}\right), \quad 1 \leq i \leq n .
$$

Because of the hypothesis (i') above, and the examples after proposition 1, each term in the chain is a differentiable family of subspaces having constant dimension. The desired basis shall be constructed by successive extensions in this chain. The key point is the application of theorem 5 in the first step, and of corollary 3 in the following ones.

First, let us see that there exists a differentiable Jordan basis of the family of restrictions $A(t) \mid: Y_{N}(t) \longrightarrow Y_{N}(t)$. Let $\bar{w}_{1}(t), \ldots, \bar{w}_{n+m}(t)$ be a differentiable basis of $\mathbb{C}^{n+m}$ such that: $\left[\bar{w}_{s+1}(t), \ldots, \bar{w}_{n}(t)\right]=Y_{N}(t),\left[\bar{w}_{1}(t), \ldots, \bar{w}_{n}(t)\right]=\mathbb{C}^{n}$, for all $t \in M$ (cf. corollary 3). Because of $A(t)\left(Y_{N}(t)\right) \subset Y_{N}(t)$ and $B(t)\left(Y_{N}(t)\right)=0$ for all $t \in M$, if we apply this change of basis to $\left(\begin{array}{l}A(t) \\ B(t)\end{array}\right)$ we obtain a matrix of the form $\left(\begin{array}{cc}\bar{A}_{11}(t) & \bar{A}_{22}^{0} \\ \bar{A}_{21}(t) & \bar{A}_{22}(t) \\ \bar{B}_{1}(t) & 0\end{array}\right)$ where, for each $t \in M, \bar{A}_{22}(t)$ is the matrix of the restriction $A(t) \mid$ in the new basis $\bar{w}_{s+1}(t), \ldots, \bar{w}_{n}(t)$. According to the hypothesis (ii'), $\bar{A}(t)$ has constant Jordan type, so that theorem 5 can be applied. Hence, there exists a differentiable Jordan basis $w_{s+1}(t), \ldots, w_{n}(t)$ of $A(t) \mid$, and the first step is finished.

Let us denote $W(t)$ the differentiable basis $w_{s+1}(t), \ldots, w_{n}(t)$ of $Y_{N}(t)$ obtained above. Let us extend this basis successively. Corollary 3 ensures the existence of a differentiable basis $V_{N-1}(t)$ such that

$$
Y_{N-1}(t)=[W(t)] \oplus\left[V_{N-1}(t)\right] .
$$


We have remarked in 3.2 that map $A(t)$ is injective on $\left[V_{N-1}(t)\right]$, and that its image forms direct sum with $Y_{N-1}(t)$ in $Y_{N-2}(t)$. Then, again by virtue of corollary 3 , there exists a differentiable basis $V_{N-2}(t)$ such that

$$
Y_{N-2}(t)=Y_{N-1}(t) \oplus\left[A(t)\left(V_{N-1}(t)\right)\right] \oplus\left[V_{N-2}(t)\right] .
$$

Following this way, the next step gives

$$
Y_{N-3}(t)=Y_{N-2}(t) \oplus\left[A^{2}(t)\left(V_{N-1}(t)\right)\right] \oplus\left[A(t)\left(V_{N-2}(t)\right)\right] \oplus\left[V_{N-3}(t)\right] .
$$

and so on. $\square$

4.5. Remarks. (1) In fact, proposition 4 can be also applied to $\bar{A}(t)$ in the above proof. Thus, in the conditions of theorem 6 , one has:

- there exist $\lambda_{1}, \ldots, \lambda_{q} \in C^{p}(M, \mathbb{C})$ such that $\lambda_{1}(t), \ldots, \lambda_{q}(t)$ are the distinct eigenvalues of $(A(t) \quad B(t))$, for every $t \in M$.

- the respective algebraic multiplicities $m_{1}, \ldots, m_{q}$ of these eigenvalues are constant.

(2) One has a similar result to the above theorem if we consider families of pairs of matrices $\left(\begin{array}{ll}A & B\end{array}\right)$ with real coefficients. The only difference is that the matrix $J(t)$ in its Brunovsky form (cf. 3.1) is a real Jordan matrix instead of a complex one. Obviously, the corresponding matrices $S(t), T(t)$ and $C(t)$ are also real.

5. Global pole assignment of class $C^{p}$. We assume that $M$ is a contractible manifold, and $(A B) \in C^{p}\left(M, M_{n \times(n+m)}(\mathbb{R})\right)$ a differentiable family of pairs of matrices having constant Brunovsky type. Then, $s=k_{1}+\ldots+k_{r}$ is constant. Also (cf. 3.5), there exist $\lambda_{1}, \ldots, \lambda_{q} \in C^{p}(M, \mathbb{C})$ such that $\lambda_{1}(t), \ldots, \lambda_{q}(t)$ are the distinct eigenvalues of $(A(t) B(t))$, having constant multiplicities $m_{1}, \ldots, m_{q}$.

We say that a set of maps $\mu_{i} \in C^{p}(M, \mathbb{C}), 1 \leq i \leq s$, is closed under conjugation if for each $i$ there is $j$ such that $\mu_{i}(t)=\overline{\mu_{j}(t)}$ for every $t \in M$.

THEOREM 5.1. Let $M$ be a contractible manifold, $(A \quad B) \in C^{p}\left(M, M_{n \times(n+m)}(\mathbb{R})\right)$ a differentiable family of pairs of matrices having constant Brunovsky type, $\lambda_{1}, \ldots, \lambda_{q} \in$ $C^{p}(M, \mathbb{C})$ giving the distinct eigenvalues of $\left(\begin{array}{ll}A & B\end{array}\right)$, and $m_{1}, \ldots, m_{q}$ their respective algebraic multiplicities. If $\mu_{i} \in C^{p}(M, \mathbb{C}), 1 \leq i \leq s$, is a set of maps closed under conjugation, then there exists a family of matrices $K \in C^{p}\left(M, M_{m \times n}(\mathbb{R})\right)$ such that the eigenvalues of $A(t)+B(t) K(t)$ are $\mu_{1}(t), \ldots, \mu_{s}(t), \lambda_{1}(t), \ldots, \lambda_{q}(t)$, the latter having multiplicities $m_{1}, \ldots, m_{q}$.

Proof. As we have said above, it is a simple adaptation, by means of theorem 6, of the proof in [9] relative to a constant pair. We enclose it for the convenience of the reader.

From (3) we know that there exists $S \in C^{p}(M, G l(n)), T \in C^{p}(M, G l(m))$, $C \in C^{p}\left(M, M_{m \times n}(\mathbb{R})\right)$ such that

$$
S(t)^{-1}(A(t) \quad B(t))\left(\begin{array}{cc}
S(t) & 0 \\
C(t) & T(t)
\end{array}\right)
$$

is a Brunovsky matrix for all $t \in M$. We denote by $(\bar{A} \bar{B})$ this Brunovsky family of matrices; that is to say

$$
\begin{aligned}
& \bar{A}(t)=S(t)^{-1}(A(t) S(t)+B(t) C(t)) \\
& \bar{B}(t)=S(t)^{-1} B(t) T(t)
\end{aligned}
$$


We shall find a family $\bar{K} \in C^{p}\left(M, M_{m \times n}(\mathbb{R})\right)$ such that $\bar{A}(t)+\bar{B}(t) \bar{K}(t)$ has the desired eigenvalues $\mu_{1}(t), \ldots, \mu_{s}(t), \lambda_{1}(t), \ldots, \lambda_{q}(t)$. Then, if we take $K(t)=C(t) S(t)^{-1}+$ $T(t) \bar{K}(t) S(t)^{-1}$, the family $A(t)+B(t) K(t)=S(t)(\bar{A}(t)+\bar{B}(t) \bar{K}(t)) S(t)^{-1}$ has the same eigenvalues. Let us construct $\bar{K}(t)$. One has

$$
(\bar{A}(t) \quad \bar{B}(t))=\left(\begin{array}{cccccccc}
N_{1} & & & & E_{1} & & & \\
& \ddots & & & & \ddots & & \\
& & N_{r} & & & & E_{r} & \\
& & & J(t) & & & & 0
\end{array}\right)
$$

(see 3.1).

Let $\ell_{j}=k_{1}+\ldots+k_{j}, 1 \leq j \leq r$ and $c_{q}^{j} \in C^{p}(M, \mathbb{C})$ defined by

$$
\left(\xi-\mu_{\ell_{j-1}+1}(t)\right) \ldots\left(\xi-\mu_{\ell_{j}}(t)\right)=\xi^{k_{j}}+\sum_{q=0}^{k_{j}-1} c_{q}^{j}(t) \xi^{q}
$$

where $\xi$ is a indeterminate. Then

$$
\left(\xi-\mu_{1}(t)\right) \ldots\left(\xi-\mu_{s}(t)\right)=\prod_{j=1}^{r}\left(\xi^{k_{j}}+\sum_{q=0}^{k_{j}-1} c_{q}^{j}(t) \xi^{q}\right)
$$

and since $\left\{\mu_{1}, \ldots, \mu_{s}\right\}$ is closed under conjugation we see that $c_{q}^{j}$ are in fact real maps.

Let $\bar{K}(t)$ the $m \times n$ matrix defined by $\bar{K}(t)=\left(\begin{array}{lllll}K_{1}(t) & K_{2}(t) \ldots K_{r}(t) & 0\end{array}\right)$ where

$$
K_{j}(t)=\left(\begin{array}{ccc} 
& 0 & \\
-c_{0}^{j}(t) & \ldots & -c_{k_{j}-1}^{j}(t) \\
0 &
\end{array}\right)(m \text {-th row })
$$

for $1 \leq j \leq r$. Then it is easy to see that the $n \times n$ matrix $\bar{A}(t)+\bar{B}(t) \bar{K}(t)$ is of the form

$$
\left(\begin{array}{cccc}
C_{1}(t) & & & \\
& \ddots & & \\
& & C_{r}(t) & \\
& & & J(t)
\end{array}\right)
$$

where

$$
C_{j}(t)=\left(\begin{array}{cccc}
0 & 1 & \ldots & 0 \\
\ldots & \ldots & \ldots & \ldots \\
0 & \ldots & 0 & 1 \\
-c_{0}^{j}(t) & \ldots & \ldots & -c_{k_{j}-1}^{j}(t)
\end{array}\right)
$$

Clearly, this matrix has the desired eigenvalues. $\square$ 


\section{REFERENCES}

[1] V.I.ARnold, On matrices depending on parameters, Usp. Math. Nauk, 26, 1971, pp. 101-114.

[2] C.I.Byrnes, On the Control of Certain Deterministic, Infinite-Dimensional Systems by Algebra-Geometric Techniques, American Journal of Math, 100 , 1978, pp. 1333-1381.

[3] L.D.Drager, R.L.Foote, C.F.Martin , Controllability of Linear Systems, Differential Geometry of Curves in Grassmannians, and Ricatti Equations, Texas Tech. University, Dept. of Mathematics 1986.

[4] J.C.Evard, J.M.Gracia, On Similarities of Class $C^{p}$ and Applications to Matrix Differential Equations, Linear Algebra Appls, 137 , 1990, pp. 363-386.

[5] J.Ferrer, I.Garca, F.Puerta, Differentiable families of subspaces, Linear Algebra Appls, 199, 1994, pp. 229-252.

[6] J.Ferrer, I.Garca, F.Puerta, Brunovsky local form of a holomorphic family of pairs of matrices, To appear in Linear Algebra Appls.

[7] J.Ferrer, F.Puerta, Similarity of non-everywhere defined linear maps, Linear Algebra Appls, 168 (1992), pp. 27-55.

[8] R.M.Guralnick, Similarity of Matrices over Commutative Rings, Linear Algebra Appls, 157 (1991), pp. 55-68.

[9] I.Gohberg, P.Lancaster, L.Rodman, "Invariant Subspaces of Matrices with Applications ", Wiley, 1986.

[10] I.GohBERG, J.LeITERER, Uber algebren stetiger operatorfunctionen, Studia Math. LVII (1976), pp. $1-26$.

[11] D.Husemoller, "Fibre Bundles ", Springer-Verlag, 1975.

[12] M.L.J.Hautus, E.D.Sontag, New results on pole-shifting for parametrized families of systems, Journal of Pure and Applied Algebra, 40 (1986), pp. 229-244.

[13] M.HAzEwinkel, (Fine) moduli (spaces) for linear systems: What are they and what are they good for, in book "Geometric Methods for the Theory of Linear Systems", edited by C.I.Byrnes and C.F.Martin, Reidel Pub. Co., Dordrecht 1980.

[14] M.Hazewinkel, A.M.Perdon, On families of systems: pointwise-local-global isomorphism problems, Int. J. Control, 33, n.4 (1981), pp. 713-726.

[15] E.D.Sontag, Linear Systems over Commutative rings: a survey, Ricerche di Automatica 7, n.1 (1976), pp. 1-34.

[16] E.D.Sontag, On Split Realizations of Reponse Maps over Rings, Information and Control, 37 (1978), pp. 23-33.

[17] E.D.Sontag, An Introduction to the Stabilization Problem for Parametrized Families of Linear Systems, Contemporary Mathematics, 47 (1985), pp. 369-400.

[18] A.Tannenbaum, "Invariance and System Theory: Algebraic and Geometric Aspects" Springer, NY, 1980.

[19] A.Tannenbaum, P.P.Khargonekar, On Weak Pole Placement of Linear Systems Depending on Parameters, "Mathematical Theory of Networks and Systems", (P.A.Fuhrmann, ed.), Springer-Verlag, Berlin, (1984), pp. 829-840.

[20] Y.Wang, E.D.Sontag, Pole Shifting for Families of Linear Systems Depending on at most three Parameters, Linear Algebra Appls, 138 (1990), pp. 3-38. 